\title{
Lectura digital en la universidad: entre lo cotidiano y lo académico
}

\author{
(Digital Reading in the University: between Every Day and Scholarly \\ Stuff)
}

\author{
Sofía Amavizca Montaño iD, Universidad Estatal de Sonora, Sonora, México \\ Erika PATricia Álvarez-Flores ' ${ }^{\circ}$, Universidad Estatal de Sonora, Sonora, México \\ DENISE HERNÁNDEZ Y HERNÁNDEZ iD Universidad Veracruqana, Veracruq, México.
}

\author{
Volumen 2, número 1 \\ Junio 2017 \\ p. $46-71$
}

Este número se publicó el 30 de junio de 2017

Artículo recibido: 16 de febrero de 2017

Artículo Aprobado: 12 de junio de 2017

ISSN: 2448-5942, doi: https://doi.org/10.36799/el.v2i1.51

\section{Cómo citar:}

Amavizca Montaño, S., Álvarez-Flores, E. P., y Hernández Y Hernández, D. (2017). Lectura digital en la universidad: entre lo cotidiano y lo académico. Estudios גambda. Teoría y práctica de la didáctica en lengua y literatura. , 2(1), 46-71. https://doi.org/10.36799/el.v2i1.51

Derechos de autor: El autor o autores conservan en todo momento sus derechos morales y patrimoniales sobre la obra; la obra no se puede alterar, transformar o ampliar; siempre debe reconocerse la autoría del documento referido. Ninguna de las modalidades de los documentos publicados en Estudios $\lambda a m b d a$. Teoría y práctica de la didáctica en lengua y literatura tienen fines comerciales de naturaleza alguna.

Los contenidos de este artículo están bajo una licencia de Creative Commons Atribución No Comercial- Sin Derivadas 4.0 Internacional (c) (1) 


\title{
Lectura digital en la universidad: entre lo cotidiano y lo académico ${ }^{1}$
}

\author{
Digital Reading in the University: between Every Day and Scholarly Stuff
}

\author{
SOFÍA AMAVIZCA MONTAÑO \\ ERIKA PATRICIA ÁlVAREZ-FLORES ${ }^{3}$ \\ DENISE HERNÁNDEZ Y HERNÁNDEZ ${ }^{4}$
}

\begin{abstract}
...Las nuevas tecnologías conllevan nuevas
formas de lectura. Hemos pasado en poco tiempo del texto ilustrado a la realidad multimedia. Mientras que los textos impresos tienen una permanencia relativa, los textos digitales son dinámicos y pueden completarse, editarse y actualizarse constantemente... (OCDE 2011 ).
\end{abstract}

\section{RESUMEN}

La inclusión de las tecnologías de información y comunicación (TIC) al entorno de la vida académica, tanto de manera formal como informal, ha modificado las prácticas de enseñanza - aprendizaje y, de manera importante, las formas de leer. La presente investigación tiene como objetivo indagar en los habitus y representaciones sociales de estudiantes universitarios respecto a la lectura, incluyendo un énfasis en la lectura en medios digitales, siendo esta una tendencia que impacta cada vez con mayor fuerza en el ámbito educativo y en las prácticas socioculturales de los universitarios. La metodología utilizada para recopilar y analizar datos fue el estudio de caso de estudiantes de dos unidades académicas de la Universidad Estatal de Sonora (UES). Como referentes teóricos se utilizaron algunos conceptos de las teorías de Pierre Bourdieu y Serge Moscovici. Los resultados demostraron que los estudiantes leen más de lo que sus profesores y ellos mismos opinan. Además, que gran parte de lo que de manera cotidiana leen, lo hacen a través de medios electrónicos y se concluye que al igual que en los medios impresos, se requiere estudiar estas formas de leer. Así como del apoyo de los docentes de las diferentes disciplinas para fortalecer las nuevas competencias lectoras de los estudiantes.

PALABRAS CLAVE: Educación superior, medios digitales, lectura, aprendizaje.

\footnotetext{
${ }^{1}$ Este artículo es producto de la Red Temática Literacidad Digital en la Universidad (RED-LDU), reconocida y aprobada por CONACyT desde mayo del 2016, conformado por investigadores, académicos y estudiantes de diversas instituciones de educación superior nacionales y del extranjero.

${ }^{2}$ Licenciada en Literaturas Hispánicas, Maestra en Educación por la Universidad del Valle de México, Doctora en Humanidades por la Universidad de Sonora. Profesora investigadora de tiempo completo en la Universidad Estatal de Sonora, Hermosillo, México. Miembro de la Red Temática Literacidad Digital en la Universidad. amavizcasofia@yahoo.com.mx

${ }^{3}$ Ingeniera Industrial en Electrónica, Doctora en Tecnologías Multimedia por la Universidad de Granada. Es Profesora Titular en Universidad Estatal de Sonora, Hermosillo, México. Sus áreas de investigación giran en torno a la utilización de tecnologías multimedia en el proceso de enseñanza-aprendizaje a nivel universitario. ericka.alvarez@ues.mx

${ }^{4}$ Doctora en Comunicación Lingüística y Mediación Multilingüe por la Universidad Pompeu Fabra, Barcelona, España. Técnico Académico del Programa de Investigación e Innovación en Educación Superior de la Universidad Veracruzana, México. Miembro de la Red Temática Literacidad Digital en la Universidad. Candidato a investigador nacional SNI. nadhernandez@uv.mx
}

Artículo recibido: 16 de febrero de 2017

Artículo enviado a corrección: 8 de mayo de 2017

Aprobado: 12 de junio de 2017

Amavižca, Álvarezy Hernández doi: https://doi.org/10.36799/el.v2i1.51 Volumen 2, número 1, Año 2017, ISSN: 2448-5942 


\begin{abstract}
The inclusion of information and communication technologies (ICT) environment on academic life, both formal and informal, has changed teaching practices - learning and, specially, ways to read. This research aims to investigate the habitus and social representations of university students about reading, including an emphasis on reading in digital media, the latter being a trend that has an increasing impact in education and the sociocultural practices in the university. The methodology used to collect and analyze data was the case study of students of two academic units of the State University of Sonora (UES). As theoretical references some concepts of the theories of Pierre Bourdieu and Serge Moscovici were used. It was observed that students read more than their teachers, so they both agree. Furthermore, most of what students read on a daily basis is through electronic media and it is to be concluded that it is necessary to also study the ways of reading on print media. This requires the support and involvement of teachers from different disciplines to strengthen the new students' reading skills.
\end{abstract}

KEYWORDS: Higher education, digital media, reading, learning.

\title{
INTRODUCCIÓN
}

Actualmente, hay una representación social, ampliamente difundida en los entornos educativos, y de manera especial en la educación universitaria, que ubican a la lectura y escritura como formas para promover en los estudiantes el desarrollo de sus procesos cognitivos para la comprensión de la ciencia y posteriormente la generación de otros saberes. Dichos procesos, se organizan y transmiten a través de los discursos orales y escritos, por tanto, leer y escribir se conciben como herramientas principales para la formación académica de los estudiantes. Pero la lectura no se circunscribe únicamente al contexto educativo, va más allá, es una práctica formal si se le ve al interior de un sistema educativo, en el currículum, las clases y las aulas. Por otro lado, la lectura también se desarrolla de manera no formal en la vida diaria, es una práctica social, tan cotidiana como: leer el periódico, un instructivo o una receta, el correo electrónico, las redes sociales, libros y revistas en formato impreso o electrónico. (Cassany 2006).

Las formas de leer están cambiando y esta situación ha generado gran cantidad de opiniones tanto a favor cómo en contra. Las voces más conservadoras aluden efectos adversos, alegando que el hecho de obtener información fácil no significa que esta sea confiable. También argumentan que a partir de la generalización de los medios digitales las personas leen menos. Por otra parte, las opiniones más vanguardistas consideran que la lectura en electrónico ofrece mayores ventajas en el aspecto educativo.

Esta investigación tiene como objetivo analizar los habitus y representaciones sociales de estudiantes universitarios respecto a la lectura, incluyendo un énfasis en la lectura en medios 
digitales, pues es una tendencia cada vez con mayor presencia en el ámbito educativo y en las prácticas socioculturales de los universitarios.

La información para este estudio se recopiló utilizando la metodología de estudio de caso con estudiantes inscritos en los semestres del segundo al cuarto de dos unidades académicas de la Universidad Estatal de Sonora (UES): la Unidad Académica Hermosillo (UAH) y la Unidad Académica Benito Juárez (UABJ). Se tomaron dichas unidades porque representan polos opuestos de la realidad institucional, una ubicada en la capital del estado con mejores condiciones para su desarrollo social y cultural y; la otra, situada en un municipio más pequeño con oportunidades más limitadas.

Los resultados demostraron que gran parte de lo que de manera cotidiana leen los jóvenes, lo hacen a través de medios electrónicos, por lo que se requiere del apoyo de los docentes de las diferentes disciplinas para fortalecer las nuevas competencias lectoras de los estudiantes en el ámbito digital.

La investigación está organizada de la siguiente manera: En la sección de aspectos teóricos se hace una revisión literaria donde se introduce a la lectura dentro del ámbito digital y se plantean las teorías de los habitus y las representaciones sociales principalmente de Pierre Bourdieu y de Serge Moscovici. Posteriormente, en la sección de metodología se describe el tipo de estudio, características de la muestra, entorno de los participantes, y los instrumentos utilizados para la medición. Se continúa con la presentación de los resultados y finalmente se hace un análisis más profundo de los resultados obtenidos, así como las referencias bibliográficas consultadas.

\section{ASPECTOS TEÓRICOS}

\section{La lectura en medios electrónicos}

La lectura digital se ha expandido a la par del internet y del uso cotidiano de los dispositivos móviles (computadoras portátiles, teléfonos móviles, tabletas y dispositivos para leer), dando paso a la hipertextualidad, al llamado "libro enriquecido" en el cual se anexan diccionarios, videos, y entrevistas con autores. Este tipo de textos y soportes demandan lectores 
con habilidades en el uso de la tecnología y les confiere una participación más activa, como coautores que da sentido y significación al texto. (Cordón, 2010).

Cordón (2010) menciona que ante la revolución digital del siglo XXI es necesario redimensionar los estudios de la lectura porque retoma espacios, situaciones y nuevas habilidades para el acceso a dichos materiales, la decodificación, la significación así como la apropiación. Gutiérrez (2006) señala que en la primera década del año 2000 el 93\% de la información que se produjo en el mundo fue en formato digital.

Por su parte, Chartier (2001) señala que la aparición del libro digital no lleva a desaparecer por completo al libro impreso, pues señala que habrá una complementariedad en la cual se establece una nueva dinámica intelectual y estética en el mundo de los libros y los lectores, para la que se requiere redefinir categorías, estéticas y legislativas que impacten en la lectura y sus actores.

La lectura en medios electrónicos confiere al lector la posibilidad de des-estructurar y reestructurar los textos originales con lo cual su trabajo de escritura puede ser más rápido y mejor. (Gutiérrez 2006). No solo eso, sino que propicia la denominada lectura de zapping, es decir poder saltar de un texto a otro o de un asunto a otro. Aunado a eso, favorece las multitareas por ejemplo: una persona puede estar realizando tareas académicas mientras chatea, escucha música o audios a la vez que realiza búsquedas en internet. (Cordón, 2010).

Los avances de la lectura en soportes digitales han sido vertiginosos, sobre todo en los países desarrollados. En el año 2009, el 53\% de la población en Francia no había escuchado hablar de los libros electrónicos pero el 70\% realizan descargas gratuitas de Internet. En 2010 en una encuesta en España sobre hábitos de lectura y compra de libros arrojó que el 48.6\% de los mayores de 14 años de este país se considera ya un lector digital, y un 76.7\% de entre los universitarios. En el 2010 los libros más vendidos en las librerías de Japón fueron creados como “novelas de pulgar”, es decir, en formato digital. (Cordón, 2010).

La inclusión de las tecnologías en las actividades editoriales hace más dinámicos los procesos de diseño, edición y difusión a la vez que se promueve el cuidado del medio ambiente en el sentido que se usa menos papel (Gutiérrez, 2006). No obstante, leer en electrónico representa un problema según Cordón (2010) por dos razones: 1) Brecha generacional para 
adultos con pocas o nulas habilidades para el uso de la tecnología, que demanda una "realfabetización" de ciudadanos para el dominio de lenguajes informáticos y diversos soportes de lectura y 2) Brecha entre países desarrollados y "países periféricos", condiciones socioeconómicas que limitan o postergan el acceso de gran parte de su población a la lectura en medios digitales.

Pese a que la lectura en medios digitales se inició hace más de veinte años en los países desarrollados, en México hasta hace pocos años ésta no era considerada como lectura propiamente por los lectores y los organismos que registraban los indicadores de lectura y ello puede deberse a diversos factores:

1) Los indicadores de lectura en México y en el contexto internacional se estimaban sobre libros impresos. Es hasta el año 2009 que la OCDE realizó su primer estudio en torno a la lectura en medios electrónicos, no obstante en esa muestra de países no estaba incluido México. Para México, dicho indicador de lectura se consideró en la Encuesta Nacional de lectura presentada por Conaculta hasta el año 2015.

2) Las instituciones de educación solo reconocían o legitimaban como leer, si los textos eran impresos y que fueran literarios o educativos. De hecho hasta la fecha los estudiantes desde el preescolar hasta la universidad se ven precisados a leer textos seleccionados por la Secretaría de Educación Pública (SEP) o por sus profesores. Por lo anterior, se puede decir que los estudiantes reproducen una representación social de que leer se refiere solo a textos impresos.

La lectura digital es una práctica sociocultural que ha cobrado gran influencia entre los estudiantes universitarios y en la sociedad en general: en sus formas de comunicarse, de leer y de educarse, por ello es necesario que las instituciones y los docentes reconozcan y apoyen el óptimo desarrollo de las competencias requeridas para esta forma de leer.

\section{Espacio social, habitus, capital cultural, capital económico y representaciones sociales}

A partir de la segunda mitad del siglo pasado, para la investigación de tipo social y humanística cobró gran relevancia utilizar metodologías diseñadas o adaptadas para observar las acciones y los percepciones de las personas, en su contexto cotidiano con sus diversas variantes, lo cual legitima y valida a este tipo de estudios. Los planteamientos de Pierre Bourdieu y de Serge Moscovici se inscriben en esa tendencia con sus teorías de los habitus y las representaciones 
sociales. De dichas propuestas se retomarán algunos conceptos para esta investigación. La lectura es una práctica social que por años fue evaluada con indicadores cuantitativos que no consideraban variables de tipo social, las cuales generan diferencias sustantivas en la interpretación de los resultados. (Moscovici 2011).

También utilizaremos el concepto de lectura desde un enfoque sociocultural, que ubica a esta actividad como construcción social, la cual se ha modificado en los diferentes momentos de la historia, la geografía y las acciones de los seres humanos. En dicha visión se ubican los diferentes tipos de textos y las diversas formas de leerlos, es decir, no hay una sola forma de leer, cada texto, disciplina y comunidad tiene sus formas específicas de leerse e interpretarse. (Cassany 2012).

Según Cassany (2006) hay tres concepciones de la comprensión lectora: 1) Concepción lingüística, donde la interpretación de un escrito es única, independientemente de las personas que lo lean y de sus condiciones al momento de hacerlo. 2) Concepción psicolingüística, la cual considera que un mismo texto se puede entender de diversas formas, dependiendo de los conocimientos y experiencias del lector virtual. 3) Concepción sociocultural, esta perspectiva refiere a que, tanto las palabras como el conocimiento previo de quien lee, se originan en la sociedad. El discurso que comparte un autor de un texto contiene sus ideas, que éste a su vez las obtuvo de otros autores y/o personas de textos o experiencias vividas. Se considera que en este último enfoque se inscribe el concepto de lectura del que se parte esta investigación y que ubica a la lectura digital como una práctica actual y cada vez más generalizada.

En este sentido, leer es una práctica cultural, un proceso comunicativo que se desarrolla en un espacio social (contexto histórico y geográfico), en el cual se decodifican e interpretan mensajes o la información de un texto escrito en forma impresa o digital que expresa ideas, pensamientos y/o sentimientos. De ahí que los profesores pretenden que los universitarios posean las habilidades y el interés por leer textos especializados en el área de su profesión, sin considerar los entornos y las variables socioculturales de los ambientes en los que se han formado.

Bourdieu se define el concepto de espacio social como el lugar en el que se ubica el objeto de estudio con sus constantes y variantes. Es un espacio en el cual confluyen y se 
relacionan seres humanos, que han sido formados por instituciones como la familia, escuela, iglesia; lo que les hace coincidir en sus prácticas y modos de pensar. En este espacio, la lectura en medios digitales cobra cada vez mayor presencia y relevancia, apoyado primero por los avances de las tecnologías de la información y la comunicación (TIC) y, posteriormente, por la cultura digital reforzada por la llamada sociedad de la información. Dicha cultura se inicia a partir de la década de los setentas, cuando las computadoras empezaron a utilizarse en las actividades de las empresas. Período en que la radio y la televisión dieron inicio a una cultura de lo audiovisual y complementó la cultura de lo impreso (Ruiz y Buira 2012).

El concepto de habitus es especialmente valioso para los estudios cualitativos de la sociedad, y Bourdieu lo define como un sistema de disposiciones duraderas que funciona como principio generador y organizador de prácticas y representaciones de las personas. Los babitus constituyen aquellas acciones que realizan las personas en la vida cotidiana y de manera consiente o no delatan o evidencian pensamientos, actitudes, creencias ante la vida (Bourdieu 1997).

Los diferentes tipos de capital aportan o limitan las condiciones de desarrollo social de los individuos, por ello han de observarse en el contexto del espacio social: El capital cultural es una cualidad de diferenciación que poseen las personas porque lo asimilaron o se los inculcaron, tiene que ver con las oportunidades o facilidades para educarse, para disponer o disfrutar de bienes culturales ${ }^{5}$ y a diferentes manifestaciones artísticas.

Según Bourdieu (1987), el capital cultural puede presentarse en tres estados:

1) El estado incorporado: refiere a disposiciones duraderas del organismo. Supone el trabajo de inculcación y de asimilación.

2) El estado objetivado: representa a la posesión de bienes culturales como: obras de arte, libros, diccionarios, instrumentos, maquinaria.

3) El estado institucionalizado: es una forma de objetivación particular como por ejemplo los títulos escolares.

\footnotetext{
${ }^{5}$ Para Bourdieu los bienes culturales a la posesión de libros, obras de arte, maquinaria entre otras cosas cuya posesión constituye la objetivación del capital cultural (Bourdieu 1987).
} 
El capital económico describe las condiciones y las posesiones de bienes materiales, así como a las capacidades de consumo de las personas y los grupos en los que se integra (Bourdieu 1987).

Para este análisis también se retoma el concepto de representaciones sociales propuesto por Moscovici, cuya teoría analiza lo que hasta ese entonces no era considerado como científico, el conocimiento de sentido común, que alude a una forma de examinar el pensamiento social. Según Moscovici las representaciones sociales son:

...una modalidad particular del conocimiento, cuya función es la elaboración de los comportamientos y la comunicación entre los individuos, es un corpus organizado de conocimientos y una de las actividades psíquicas gracias a las cuales los hombres hacen inteligible la realidad física y social, se integran en un grupo o en una relación cotidiana de intercambios ... (Moscovici 1979:18).

El concepto de representaciones sociales constituye una rama del conocimiento para investigar y organizar los descubrimientos en la realidad social. La representación social es un proceso que hace que el concepto y la percepción sean intercambiables porque se generan de manera bilateral (Moscovici 2011).

Un aspecto muy importante en la práctica de la lectura es la participación del lector, pues es él quien da sentido y significación a lo establecido por el autor. En este proceso el autor es un creador y el receptor o lector se convierte en un co-creador.

La teoría de la recepción de Roman Ingarden ofrece una importante aportación, la concretización de los espacios indeterminados en el texto, que son menores en la obra científica que en la literaria, pues esta última necesita de la co-creación y parte de la experiencia estética proporcionada por el autor y recibida por el lector (Ingarden, 2005). Este teórico señala que la comprensión constituye solo una de las formas en las que el lector puede relacionarse con la obra. Alude también que la aproximación del lector hacia el texto se realiza de formas diferentes, dependiendo del tipo de obra que se lea, las actitudes hacia dicho texto conducen al conocimiento del mismo.

Amavižca, Álvarezy Hernández doi: https://doi.org/10.36799/el.v2i1.51 Volumen 2, número 1, Año 2017, ISSN: $2448-5942$ 
La descripción de los procesos cognoscitivos involucrados en la lectura de un texto, en su desarrollo y su carácter específicos, y juzgamos si son positivamente efectivos, esto nos puede conducir a un conocimiento objetivamente válido de la obra. (Ingarden, 2005, p.16)

Según Ingarden (2005) la lectura es una actividad de percepción sensorial en la que se transmiten cualidades emocionales, signos verbales, sonidos, significaciones que dan sentido a lo que se lee, por ello la comprensión se da de manera entrelazada. La comprensión lectora se compone de procesos heterogéneos y de procesos temporales.

Refiere que hay dos tipos de lecturas: una, la ordinaria o pasiva, que es más mecánica o superficial. La otra, es la activa en la cual se pone atención, se construye un sentido y una objetivación del texto.

En la teoría de Ingarden (2005) se establece el concepto de "indeterminación" que se refiere a los aspectos del objeto representado que no están en ella. Dichos aspectos no están presentes en la obra por casualidad o por un error del escritor, faltan porque, dada la estructura de la obra, esta no puede determinar todo, pero en el proceso de recepción que este teórico llama concreción se fijan por el lector.

A partir de las ideas expresadas en la teoría de la recepción de Ingarden es posible comprender que en el proceso de la recepción de la lectura ocupa un papel preponderante el lector, quien da orden y sentido a lo indeterminado de un texto y es esa parte activa, con sus marcos de referencia, lo que enriquece la interpretación de la obra. Esta teoría es relevante para esta investigación ya que ubica a la lectura como un acto de conciencia individual, subjetiva en el cual intervienen variables personales y sociales.

También contribuye a dar flexibilidad al concepto de lectura y de lector, en el sentido que hay una gran variedad de textos, los cuales demandan diferentes formas de leer. Por lo mismo hay también una gran cantidad de tipos de lectores dependiendo de sus preferencias en temas, tipos de textos o de soportes.

\section{La institucionalización de la lectura digital}

La generalización de la lectura digital en la UES ha sido apoyada por la institución. En el año 2008 se adquirió la primera base de datos (E-Libro), que en ese entonces contaba con 18 
mil textos (entre libros, artículos de revistas, tesis y documentos en general). Actualmente, en la institución se tienen 54 bases de datos, con 125,251 documentos electrónicos entre libros, artículos y otros documentos para consultas (UES 2016).

La adquisición de esta base de datos se hizo debido a sus características y su sencillez para realizar consultas (no se requiere tener experiencia en el uso de estas), y que gran parte de sus textos son de interés para alumnos y profesores de los programas educativos con mayor matrícula en la institución (las áreas económico administrativas) e inclusive porque contienen un alto porcentaje de bibliografía en español.

\section{METODOLOGÍA}

Se considera para esta investigación el estudio tipo caso como más propicio para realizar una aproximación social a las acciones cotidianas relacionadas a las prácticas de la lectura de los estudiantes universitarios, en un contexto social específico y en un momento histórico actual (Martínez 2006). A través de este estudio, es posible analizar las diversas aristas que proporciona ubicar los habitus y las representaciones de la lectura en los estudiantes de la UES, en un espacio social, considerando aspectos como el capital cultural y el económico, expresados en la teoría de Bourdieu.

\section{Población}

Esta metodología permite profundizar en las representaciones sociales y analizar las opiniones de los estudiantes y cómo dichas representaciones se adquieren y se reproducen en el contexto sociocultural.

Se escogieron para la investigación las unidades académicas de Hermosillo y de Benito Juárez por las condiciones socioculturales que se presentan en ellas, muy diversas o distintivas respecto al resto de las unidades académicas. La Unidad Académica Hermosillo se ubica en la capital del estado de Sonora, es la que cuenta con mayor población, y posee servicios e infraestructura educativa y cultural de una ciudad desarrollada. Por su parte, la Unidad Académica Benito Juárez, ubicada en una población pequeña, enclavada en una zona de actividades económicas y condiciones rurales.

Amavižca, Álvarezy Hernández doi: https://doi.org/10.36799/el.v2i1.51 Volumen 2, número 1, Año 2017, ISSN: $2448-5942$ 


\section{Instrumentos y muestra}

Los instrumentos que se utilizaron fueron la encuesta y grupos focales. Para determinar el tamaño de la muestra y que esta fuera representativa de la población de estudiantes de la UES, y con el grado de confiabilidad aceptable para un estudio, se consideró la siguiente fórmula (Hernández, Fernandez y Baptista 2006):

$$
n=\frac{N * Z^{2} * p^{*} q}{d^{2} *(N-1)+Z^{2} * p^{*} q}
$$

$\mathrm{N}=$ se refiere al total de alumnos inscritos en la UES

$\mathrm{Z}=$ Es el intervalo de confianza (para esta investigación se utiliza el 95\%)

$\mathrm{P}=$ Proporción esperada

$\mathrm{q}=1-\mathrm{p}(50 \%)$

$\mathrm{d}=$ tamaño del error (se considera $6 \%$ )

La muestra se integró por 445 estudiantes de las dos unidades académicas (281 de la UAH y 164 de la UABJ), estudiantes inscritos en los semestres del segundo al cuarto.

La encuesta se realizó con la aplicación de un cuestionario integrado por 31 preguntas cerradas y 12 preguntas abiertas. Para propósito de esta investigación se considera como pregunta cerrada la que da varias opciones de respuesta al encuestado y las preguntas abiertas son las se orientan a conocer de manera libre, la opinión o la información que proporciona quien responde dicha encuesta.

La entrevista se realizó a dos grupos focales (GF) uno en la UABJ constituido por 18 estudiantes y otro por 10 estudiantes de la UAH. En ambos grupos participaron estudiantes de todos los programas educativos. El entrevistador dirigió 19 preguntas abiertas, orientadas a conocer la opinión de los participantes y los habitus relacionados con la lectura.

Por los instrumentos utilizados y la interpretación que se da a la información recabada es posible expresar que esta investigación es mixta, ya que se utilizan instrumentos cualitativos y cuantitativos, aunque, finalmente, por la naturaleza social del objeto de estudio, la interpretación de la información es más cualitativa. 


\section{RESULTADOS}

\section{Entorno Social y Económico de Estudiantes}

Para analizar las prácticas lectoras, en cualquiera de sus variantes, es importante ubicar a dichas prácticas en un entorno social, lo que en términos de Bourdieu es el espacio social en el que confluye el capital cultural, social y económico.

Los resultados expuestos en relación al entorno social y económico en general de los alumnos participantes indican que el 51\% pertenecen a la clase media baja, el 26\% a la clase baja y el $12.5 \%$ a la clase más baja. Lo cual confirma lo que constituía un supuesto de esta investigación: Los estudiantes que ingresan a la UES son jóvenes que provienen de escuelas y entornos familiares con menos condiciones favorables para su desarrollo académico que los que pertenecen a la clase alta. La Figura 1, muestra los índices de estudiantes por unidad académica según su nivel socioeconómico.

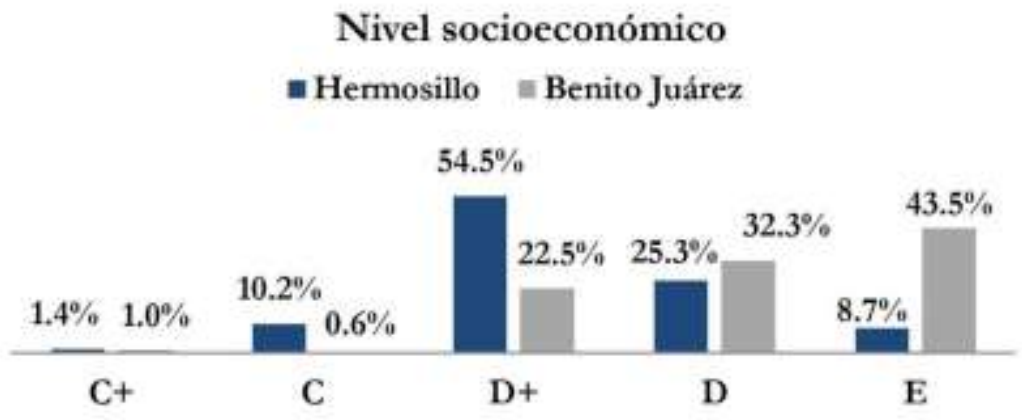

Figura 1. Nivel socioeconómico por unidad académica Elaboración propia a partir de encuesta aplicada para este estudio, 2014. Se consideró para ello la regla 7X7 del AMAI. ${ }^{6}$

${ }^{6}$ El nivel socioeconómico se determinó con la regla AMAI NSE 8X7, utilizada para generar los indicadores oficiales como los del Instituto Nacional de Estadística, Geografía e Informática (INEGI).

\begin{tabular}{|l|l|}
\hline $\begin{array}{l}\text { NIVEL } \\
\text { SOCIOECONÓMICO }\end{array}$ & $\begin{array}{l}\text { RANGO DE INGRESO } \\
\text { MENSUAL FAMILIAR }\end{array}$ \\
\hline A/B Clase alta & $\$ 85,000.00$ ó más \\
\hline C + Clase media alta & $\$ 35,000.00$ a $\$ 84,999.00$ \\
\hline C Clase media & $\$ 11,600.00$ a $\$ 34,999.00$ \\
\hline D+ Clase media baja & $\$ 6,800.00$ a $\$ 11,599.00$ \\
\hline D Clase baja & $\$ 2,700.00$ a $\$ 6,799.00$ \\
\hline E Clase más baja & $\$ 0.00$ a $\$ 2,699.00$ \\
\hline
\end{tabular}

Amavižca, Álvarezy Hernández doi: https://doi.org/10.36799/el.v2i1.51 Volumen 2, número 1, Año 2017, ISSN: 2448-5942 
Analizando el entorno socio-cultural, se observó que solo el $24.5 \%$ de los padres y el $23 \%$ de las madres de los estudiantes participantes de la UAH tenían estudios profesionales. Para el caso de UABJ, los índices son $9.6 \%$ de los padres y el $11.5 \%$ de las madres con estudios profesionales.

Las condiciones socioeconómicas de los estudiantes son importantes para analizar su capital cultural, pues no podemos desvincular la vida, el entorno de los habitus y de sus sistemas de creencias, precisamente ese es el error en el que se incurre cuando se analizan solo los números duros de los indicadores de lectura, al respecto expresa Juan Domingo Argüelles:

... la lectura tiene que ver con asuntos estructurales donde los problemas socioeconómicos son más apremiantes que la lectura. Cuando algunos medios aprendan a leer en la realidad y en los libros, entenderán por qué nuestros índices de lectura no se parecen a los de Finlandia (Domingo, 2010,58).

Se observó que la actividad principal de los padres de los estudiantes encuestados es la de "empleado", para el caso de las madres, el 52 \% de la Unidad Académica Benito Juárez (UABJ) se dedican al hogar, en este mismo ámbito se encuentra el $45 \%$ de las madres de los estudiantes de la Unidad Académica Hermosillo (UAH).

\section{Habitus de Lectura}

Los aspectos socioculturales de las familias de los estudiantes les generan el ambiente en el que pueden desarrollarse o no hábitos de lectura, en cualquiera de los soportes impresos o digitales. Tal como se observa en la Figura 2, menos del 50\% de los estudiantes encuestados se conciben como lectores. Sin embargo, en esta Figura 2, se observa que, contrario a lo esperado, por las condiciones socioculturales del municipio Benito Juárez, los estudiantes de la UABJ estos manifiestan tener un porcentaje superior con hábitos de lectura. En sentido, cabe mencionar que se observó en esta unidad académica labor de los profesores que imparten asignaturas relacionadas a la lectura ha establecido importantes diferencias. Los estudiantes en las encuestas y, de manera especial en los grupos focales, señalaron que les gustaba leer, así como las clases de fomento a la lectura. Expresaron que las dinámicas de las clases y lo textos ahí analizados les parecían interesantes y les ayudaban a fortalecer su gusto por leer y su capacidad de análisis.

Amavižca, Álvarezy Hernández doi: https://doi.org/10.36799/el.v2i1.51 Volumen 2, número 1, Año 2017, ISSN: $2448-5942$ 


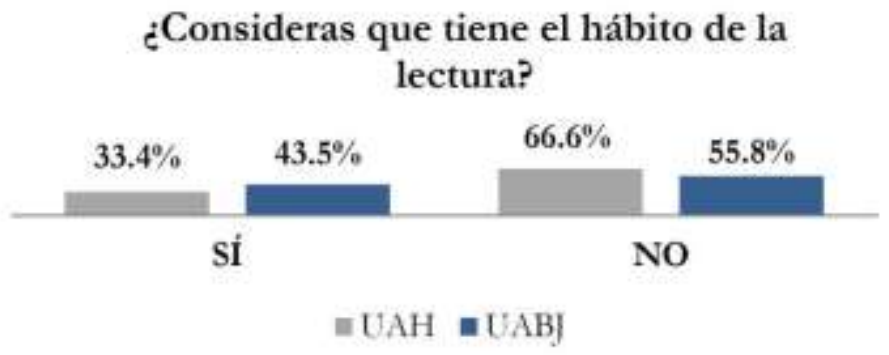

Figura 2. Índice de alumnos respecto al hábito de leer. Elaboración propia a partir de encuesta, 2014.

En la actualidad, en la llamada sociedad del conocimiento, el fácil acceso a los contenidos disponibles en internet y la aparición de otros medios electrónicos ha llevado a un cambio en la dinámica de los procesos de aprendizaje y comunicación, sobre todo entre los jóvenes. De ahí que dentro de los habitus o prácticas relacionadas con la lectura están evolucionando de manera constante y sustancial. Es decir, que las formas de leer y de aprender están cambiando. La tendencia para leer cada es más común hacerlo en formato electrónico y en diversos tipos de dispositivos móviles debido a que es más práctico y económico. También cada vez se generaliza más la utilización de plataformas educativas y una serie de recursos digitales de apoyo para el aprendizaje.

La lectura ya no se no se limita a documentos impresos o en la computadora, incluye además el teléfono celular, mensajes por correo electrónico, entre otros. Se está presentando un nuevo escenario del proceso de lectura y con ello se visualiza un nuevo protagonista de éste. Además, se pone de manifiesto la eminente incorporación de la lectura digital entre los estudiantes, no solo para el ámbito académico, el 55.10\% de los jóvenes entrevistados demuestran que están inmersos en el uso de las tecnologías digitales como por ejemplo para descargar de Internet e imprimir documentos web de otra índole, visitas a foros, blogs y la utilización de otros tipos de servicios en la denominada "nube". Para lo cual, al menos pasan 1 a 2 horas al día utilizando la computadora, incluso un $10.50 \%$ de estudiantes utilizan la computadora por más de 5 horas al día.

Tal como se representa en la Tabla 1 y Tabla 2, todos llevan a diario prácticas de lecturas de origen digital. Esto se manifiesta en la utilización de redes sociales, correo electrónico y por 
ver y/o bajar videos de You Tube. Además de considerar durante su navegación por Internet de otro tipo de páginas web en general. Como se puede observar las redes sociales tienen preferencia entre los estudiantes encuestados en ambas unidades académicas.

\begin{tabular}{|c|c|c|c|c|c|}
\hline \hline \multirow{2}{*}{$\begin{array}{c}\text { Tiempo que utilizan la } \\
\text { computadora al día. }\end{array}$} & \multicolumn{5}{|c|}{ Tipo de consultas principalmente en la WEB o Internet } \\
\cline { 2 - 6 } UAH & $\begin{array}{c}\text { Redes } \\
\text { sociales }\end{array}$ & $\begin{array}{c}\text { Correo } \\
\text { electrónico }\end{array}$ & You tube & $\begin{array}{c}\text { Páginas } \\
\text { diversas }\end{array}$ & Nc \\
\hline 1 a 2 hrs. & $57.7 \%$ & $9.2 \%$ & $3.8 \%$ & $29.2 \%$ & $0.0 \%$ \\
3 a 4 hrs. & $56.3 \%$ & $6.3 \%$ & $5.4 \%$ & $31.3 \%$ & $0.7 \%$ \\
Más de 5 hrs. & $44.4 \%$ & $5.6 \%$ & $5.6 \%$ & $44.4 \%$ & $0.0 \%$ \\
\hline \hline
\end{tabular}

Tabla 1. Tipo de consultas principalmente en la WEB o Internet de estudiantes UAH según el tiempo utilizado al día.

\begin{tabular}{|c|c|c|c|c|c|}
\hline \hline \multirow{2}{*}{$\begin{array}{c}\text { Tiempo que utilizan la } \\
\text { computadora al día. }\end{array}$} & \multicolumn{5}{|c|}{ Tipo de consultas principalmente en la WEB o Internet } \\
\cline { 2 - 6 } UABJ & $\begin{array}{c}\text { Redes } \\
\text { sociales }\end{array}$ & $\begin{array}{c}\text { Correo } \\
\text { electrónico }\end{array}$ & You tube & $\begin{array}{c}\text { Páginas } \\
\text { diversas }\end{array}$ & Nc \\
\hline 1 a 2 hrs. & $50.0 \%$ & $17.7 \%$ & $4.2 \%$ & $27.1 \%$ & $1.0 \%$ \\
3 a 4 hrs. & $80.8 \%$ & $7.7 \%$ & $0.0 \%$ & $11.5 \%$ & $0.0 \%$ \\
Más de 5 hrs. & $42.9 \%$ & $0.0 \%$ & $0.0 \%$ & $57.1 \%$ & $0.0 \%$ \\
\hline \hline
\end{tabular}

Tabla 2. Tipo de consultas principalmente en la WEB o Internet de estudiantes UABJ según el tiempo utilizado al día.

En consecuencia, dentro de los resultados expuestos del estudio se perciben habitus lectores relacionados con la lectura digital en sus actividades diarias. Cabe mencionar que los estudiantes que participaron en dicho estudio tienen entre 18 y 23 años, edad que los ubica entre los nativos digitales, una generación en la cual es cotidiano el uso de las computadoras y otros dispositivos digitales. Muchos de esos jóvenes son usuarios del Internet y las computadoras desde niños. 


\begin{tabular}{|c|c|c|c|c|c|c|}
\hline \multirow{2}{*}{$\begin{array}{l}\text { Hábito de } \\
\text { lectura }\end{array}$} & \multirow[b]{2}{*}{$\begin{array}{c}\text { Unidad } \\
\text { Académica }\end{array}$} & \multicolumn{5}{|c|}{ Medios que prefieren utilizar para lee } \\
\hline & & Internet & $\begin{array}{l}\text { Libro } \\
\text { tradicional }\end{array}$ & $\begin{array}{l}\text { Audio } \\
\text { libros }\end{array}$ & $\begin{array}{l}\text { Libros } \\
\text { electrónicos }\end{array}$ & $\mathrm{Nc}$ \\
\hline & UAH & $10.5 \%$ & $73.7 \%$ & $1.1 \%$ & $14.7 \%$ & $0.0 \%$ \\
\hline & UABJ & $37.3 \%$ & $33.9 \%$ & $1.7 \%$ & $27.1 \%$ & $0.0 \%$ \\
\hline \multicolumn{2}{|c|}{$\begin{array}{c}\text { Total con hábito de Lectura } \\
\text { según medio }\end{array}$} & $20.8 \%$ & $58.4 \%$ & $1.3 \%$ & $19.5 \%$ & $0.0 \%$ \\
\hline No & $\mathrm{UAH}$ & $43.2 \%$ & $47.6 \%$ & $1.6 \%$ & $7.0 \%$ & $0.5 \%$ \\
\hline & $\mathrm{UABJ}$ & $54.3 \%$ & $27.1 \%$ & $1.4 \%$ & $15.7 \%$ & $1.4 \%$ \\
\hline \multicolumn{2}{|c|}{$\begin{array}{l}\text { Total sin hábito de Lectura } \\
\text { según medio }\end{array}$} & $46.3 \%$ & $42.0 \%$ & $1.6 \%$ & $9.4 \%$ & $0.8 \%$ \\
\hline \multicolumn{2}{|c|}{ Total según medio utilizado } & $36.6 \%$ & $48.3 \%$ & $1.5 \%$ & $13.2 \%$ & $0.5 \%$ \\
\hline
\end{tabular}

Tabla 3. Medios que prefieren utilizar para leer según su hábito de Lectura.

Los dispositivos de lectura digital cada vez tienen mayor presencia entre los estudiantes que se inclinan a la práctica de la lectura, donde el 19.50\% prefieren los libros electrónicos como medio para leer y el 20.8\% prefieren el Internet. Entre los estudiantes que expresan "no tienen el hábito de lectura" ya predomina un $55.7 \%$ que prefiere utilizar medios digitales para leer (Internet y Libros electrónicos). Lo anterior nos revela que aunque los jóvenes no se asumen como lectores, si leen, pero no tanto de la forma tradicional (impreso). Esto nos presenta, tanto para la UAH como para UABJ, a un tipo de lectores digitales, es decir que el desarrollo de la tecnología incide en las formas de leer y de comunicarse de la sociedad (Ver Tabla 3).

Estas formas de leer establecen nuevas prácticas lectoras y comunicarse con códigos propios de estos medios que los estudiantes reconocen y decodifican cumpliendo con lo que expresa Ingarden (2005) pues se instituyen como lectores que dan sentido a lo que leen como coautores, que determinan lo indeterminado de los textos, les permite acceder y compartir información con oportunidad y flexibilidad, desde una computadora hasta los dispositivos móviles. 
Leer en estos medios proporciona una significación especial al hecho de concebirse como lector, pues en el universo casi ilimitado de leer en internet o en medios electrónicos permite a los estudiantes acceder a todo tipo de textos de su interés que les hace ser más participativos, autónomos y rápidos en el acceso a la información.

En el análisis se percibe un $51.3 \%$ de estudiantes que prefieren los medios digitales, independientemente si poseen hábitos de lectura o no, antes que recurrir al libro tradicional (con tan solo el 48.30\%). Es importante denotar en la Tabla 3 que hay un mayor porcentaje de estudiantes de la UABJ con respecto a la UAH que prefieren el ámbito digital que el tradicional, independientemente de su práctica Lectora.

Según el estudio, de los encuestados que declaran tener hábitos de lectura, un 64.9\% lo hace por gusto y un 31.8\% lo hace tanto por gusto como por obligación, se destaca que el 66\% de la lectura por placer sigue siendo a través del libro tradicional. Con este último dato se puede observar que, pese a lo generalizado de los medios electrónicos para leer, los estudiantes comparten aún la representación social de que leer por placer o leer verdaderamente solo se hace si se utilizan libros impresos.

En términos de Ingarden (2005) leer en medios digitales tiene una significación poco académica para los estudiantes, ya que ellos utilizan de manera cotidiana y desde niños, como medio de comunicación y de expresión. En este sentido ellos son lectores activos que interpretan y comparten, no solo el lenguaje escrito sino también imágenes, videos, sonidos; códigos propios de la comunicación en estos soportes y en procesos heterogéneos y temporales. La significación de ser lector en estos soportes no solo abarca los textos, sino también a los mismos lectores quienes comparten aún una representación social de que ser lector es leer libros impresos, no tanto en medios electrónicos. Por lo anterior, según Ingarden en concebirse o no como lector reside en el proceso de recepción, pues constituye un conector entre el texto y el lector.

\section{Lectora con Propósitos Académicos}

Leer en medios electrónicos actualmente abarca los dos tipos de lectura referidos por Ingarden (2005) la ordinaria que se ha convertido en cotidiana (resultados vistos en Tabla 1, Tabla 2 y Tabla 3), la utilizada todos los días para comunicarse e informarse tanto en medios 
electrónicos y dispositivos móviles. La otra, no menos activa, se orienta a construir un sentido y una objetivación del texto que puede ser en muchos casos para propósitos académicos.

A este respecto, en la investigación se aprecia que un porcentaje importante de estudiantes declaran leer por obligación diversos materiales, es decir que se ven precisados a leer en el ámbito digital o fuera de él, por razones académicas. Y tal como se aprecia en Tabla 4, el $29.2 \%$ de los estudiantes de UABJ se ven obligados a leer en medios digitales (Textos de Internet) a diferencia de un $26.4 \%$ de los jóvenes de UAH.

\begin{tabular}{|c|c|c|c|c|c|c|}
\hline \hline \multirow{2}{*}{$\begin{array}{c}\text { Unidad } \\
\text { Académica }\end{array}$} & \multicolumn{6}{|c|}{ Tipo de Lectura que leen por obligación } \\
\cline { 2 - 7 } & $\begin{array}{c}\text { Libros de texto } \\
\text { académico }\end{array}$ & $\begin{array}{c}\text { Periódicos } \\
\text { y/o revistas }\end{array}$ & $\begin{array}{c}\text { Textos de } \\
\text { Internet }\end{array}$ & Biografías & Otro & Nc \\
\hline UAH & $39.3 \%$ & $9.3 \%$ & $26.4 \%$ & $5.4 \%$ & $2.5 \%$ & $17.1 \%$ \\
UABJ & $36.2 \%$ & $22.3 \%$ & $29.2 \%$ & $3.3 \%$ & $0.0 \%$ & $10.0 \%$ \\
Total & $38.3 \%$ & $13.4 \%$ & $27.3 \%$ & $4.4 \%$ & $1.7 \%$ & $14.9 \%$ \\
\hline \hline
\end{tabular}

Tabla 4. Tipo de Lectura que leen por obligación según Unidad Académica.

Aunado a esto, los resultados de este estudio, obtenidos mediante la encuesta y los grupos focales, ponen de manifiesto que para el $89.50 \%$ de los estudiantes el internet es un recurso altamente valorado para hacer las tareas académicas. De los estudiantes que dicen tener el hábito de la lectura, un $64.3 \%$ de ellos utilizan el Internet para hacer sus tareas y un 18.2\% los libros electrónicos. Aumentando el porcentaje de utilización de Internet (a 83.5\%) para aquellos que no tienen hábito de lectura. Al hacer el análisis según la unidad académica a la que están adscritos, se visualiza que el porcentaje de utilización en el ámbito digital o fuera de él es muy similar, tal como se aprecia en la Tabla 5. 


\begin{tabular}{|c|c|c|c|c|}
\hline \hline \multirow{2}{*}{$\begin{array}{c}\text { Unidad } \\
\text { Académica }\end{array}$} & \multicolumn{4}{|c|}{ Fuente utilizada con mayor frecuencia para hacer tareas } \\
\cline { 2 - 5 } & Internet & $\begin{array}{c}\text { Libros } \\
\text { impresos }\end{array}$ & $\begin{array}{c}\text { Libros } \\
\text { electrónicos }\end{array}$ & $\begin{array}{c}\text { Revistas y } \\
\text { periódicos }\end{array}$ \\
\hline UAH & $76.1 \%$ & $10.4 \%$ & $13.2 \%$ & $0.4 \%$ \\
UABJ & $76.2 \%$ & $8.5 \%$ & $13.8 \%$ & $1.5 \%$ \\
Total & $76.1 \%$ & $9.8 \%$ & $13.4 \%$ & $0.7 \%$ \\
\hline \hline
\end{tabular}

Tabla 5. Fuente utilizada con mayor frecuencia para hacer tareas según Unidad Académica.

En este sentido, puede visualizarse en los resultados claramente cómo se presenta un lector digital que da respuesta a las tecnologías de la información y comunicación, y por ende a sus nuevos entornos sociales y tecnológicos, independientemente de sus hábitos de lectura y de su entorno socioeconómico. Lo que en términos de Bourdieu quiere decir que los estudiantes y la sociedad poseen un capital cultural objetivado, ahora en bienes culturales como lo son las computadoras, dispositivos móviles y otros elementos digitales (el internet o medios multimedia).

Respaldando los resultados el hecho de que un $87.30 \%$ de los estudiantes prefieren el Internet y los libros electrónicos como medios para hacer tareas o investigaciones antes de utilizar un libro tradicional, independientemente de si tienen hábitos de lectura o no. Esto nos presenta un capital cultural en su estado incorporado, pues cada vez se asimila más la lectura en medios digitales.

Un punto a resaltar es que es ligeramente más alto el porcentaje de estudiantes de la UABJ con respecto a los de UAH que gustan de utilizar el Internet (Ver Tabla 6), no obstante tener un nivel socioeconómico más bajo y que pudiesen tener menos acceso a este medio. 


\begin{tabular}{|c|c|c|c|c|}
\hline \multirow{2}{*}{$\begin{array}{c}\text { Unidad } \\
\text { Académica }\end{array}$} & \multicolumn{4}{|c|}{ Medios que prefieren utilizar para hacer tareas o investigaciones } \\
\cline { 2 - 5 } & Internet & Libro tradicional & Libros electrónicos & $\mathrm{Nc}$ \\
\hline UAH & $75.7 \%$ & $13.9 \%$ & $10.0 \%$ & $0.4 \%$ \\
UABJ & $79.2 \%$ & $8.5 \%$ & $11.5 \%$ & $0.8 \%$ \\
Total & $76.8 \%$ & $12.2 \%$ & $10.5 \%$ & $0.5 \%$ \\
\hline \hline
\end{tabular}

Tabla 6. Medios que prefieren utilizar para hacer tareas o investigaciones según Unidad Académica.

Por otro lado, los resultados indican que el $81.2 \%$ de quienes no consideran tener el hábito de la lectura prefieren el Internet como medio de consulta para hacer tareas o investigaciones, es decir, prefieren leer en medios electrónicos por lo cual podemos interpretar que tienen una representación social de lo que es "ser lector" o tener el hábito de la lectura, en leer libros o textos impresos.

Se observa que los estudiantes manejan dos conceptos de lectura, uno "oficial" o institucionalizado, que corresponde al canon establecido en el ámbito académico, que ha sido inculcado en la sociedad por medio de las escuelas y las instituciones de difusión cultural. La otra, una lectura "más cotidiana o práctica", la que se hace de placer o por necesidad de mantenerse informado, ya sea en medios electrónicos como las redes sociales y en los libros que a ellos les gustan de superación personal, de literatura y de temas diversos, que académicos o no, son del interés de los lectores.

Asimismo, en el análisis de los comentarios hechos por los estudiantes en los grupos focales, se pudo observar que ellos, pese a que sí leen y les gusta hacerlo, no se consideran lectores, ni asumen que sus compañeros lo sean, pese a que leen por horas en medios digitales. Con ello ubicamos nuevamente que aún persiste la representación social de que la lectura, para considerarse como tal "debe de ser" de textos legitimados por alguna autoridad académica o editorial. Es decir, se ubica a la lectura o se hace una separación de lo que es la lectura como una práctica social más académica que cotidiana. De acuerdo a la teoría de Ingarden (2005) no debiera haber una separación tan tajante ya que en cualquier texto y soporte el lector da sentido a lo indeterminado del texto por medio de códigos comunes.

Amavižca, Álvarezy Hernández doi: https://doi.org/10.36799/el.v2i1.51 Volumen 2, número 1, Año 2017, ISSN: $2448-5942$ 
La institucionalización de la lectura digital ha permitido en los últimos años que en la UES se haya incrementado la lectura de textos en bases de datos. No obstante, tal como se muestra en la Tabla 7, los estudiantes pese a que son nativos digitales, no son usuarios asiduos de bases de datos como fuentes más confiables; más bien prefieren leer en sitios de acceso abierto en internet y se comunican por medio de las redes sociales; consultan páginas de internet, blogs y redes sociales. Esto quizás, debido a que estas herramientas no especializadas tiene gran diversidad temática, son para ellos más sencillas y rápidas de leerles y además; les demandan períodos de atención más cortos que en las bases de datos. Propiciando que haya más lectores por otros medios no académicos.

\begin{tabular}{|c|c|c|c|c|c|c|c|}
\hline Año & $\begin{array}{c}\text { Páginas } \\
\text { consultadas }\end{array}$ & $\begin{array}{c}\text { Páginas } \\
\text { copiadas }\end{array}$ & $\begin{array}{c}\text { Páginas } \\
\text { impresas }\end{array}$ & Títulos & $\begin{array}{c}\text { Sesiones } \\
\text { por } \\
\text { usuario }\end{array}$ & $\begin{array}{c}\text { Descargas } \\
\text { capítulo/Página }\end{array}$ & $\begin{array}{c}\text { Descargas } \\
\text { título } \\
\text { completo }\end{array}$ \\
\hline 2012 & 86,801 & 1,496 & 1,296 & 4,031 & 6,906 & 78 & 50 \\
\hline 2013 & 101,612 & 2,033 & 627 & 4,870 & 8,523 & 207 & 146 \\
\hline 2014 & 144,557 & 2,915 & 446 & 5,434 & 10,606 & 987 & 259 \\
\hline 2015 & 228,351 & 7,251 & 12,292 & 8,568 & 15,720 & 468 & \\
\hline
\end{tabular}

Tabla 7. Estadísticas de uso de E-Libro en la UES del 2012 al 2014. Información tomada del documento de Estadísticas anuales de biblioteca UES.

Como se puede observar en la misma tabla, la utilización de E- libro se incrementó de manera paulatina con el paso de los años, aunque el personal bibliotecario ha considerado que dichos recursos aún están siendo subutilizados. Lo mismo puede apreciarse en las Tablas 8 y 9 donde se muestra la utilización de otras dos bases de datos (Conricyt y Springer E-Book).

En términos de Bourdieu (1987) las bases de datos, las plataformas educativas y los dispositivos móviles constituyen parte del capital cultural objetivado al que acceden los estudiantes en la UES, pues ya no solo disponen de libros impresos en las bibliotecas, también cuentan con una biblioteca digital a la cual pueden ingresar desde cualquier sitio o dispositivo con conexión a Internet. 
Sin embargo, en los grupos focales los estudiantes expresaron que pese a lo generalizado de la lectura en medios electrónicos y digitales ello no garantiza que se lea mejor o más, pues, a decir de ellos mismos, persisten entre los estudiantes las prácticas de copiar y pegar de internet sin leer o analizar con atención los textos para poderlos interpretar de manera adecuada.

Se observa también como los estudiantes reconocen en la lectura en medios electrónicos a una forma de comunicarse e informarse, pero que aún no han transitado tanto a ser lectores asiduos de documentos en bases de datos, cuyo respaldo académico supone que se trata de textos académicos que aportarían más a la formación profesional de los estudiantes.

\begin{tabular}{|c|c|c|c|c|}
\hline CONRICYT & 2011 & 2012 & 2013 & 2014 \\
\hline TOTAL & 20 & 1,234 & 1,537 & 2,626 \\
\hline
\end{tabular}

Tabla 8. Estadísticas de uso de bases de datos de Conricyt en la UES del 2011 al 2013 . Información tomada del documento de Estadísticas anuales de biblioteca UES.

\begin{tabular}{|c|c|}
\hline Año & Consultas Springer E-books \\
\hline 2013 & 993 \\
\hline 2014 & 3,783 \\
\hline 2015 & 1,940 \\
\hline
\end{tabular}

Tabla 9. Estadísticas de uso de bases de datos de Springer E-Books en la UES del 2013 al 2014. Información tomada del documento de Estadísticas anuales de biblioteca UES.

Aunado al material para consulta disponible a través de la biblioteca digital institucional, la UES pone a disposición de los estudiantes otros recursos web como correo, blogs, portales y a partir del 2014, una plataforma académica que apoya significativamente al proceso de enseñanza- aprendizaje en todos sus programas académicos; mediante la cual el estudiante lleva a cabo de forma obligatoria actividades en forma virtual de las diversas asignaturas en las que está inscrito. Adicional a esas actividades, los estudiantes tienen acceso a material de apoyo, notificaciones, entre otro tipo de información referente al curso. Propiciando que se lleve a cabo una práctica social "formal” del proceso de lectura digital, aún que esto no sea percibido como tal. 
En definitiva, las plataformas educativas, los libros y archivos digitales han dado un impulso a nuevas formas de lectura, promoviendo con ello la alfabetización y mejora de la calidad de vida en general. Sin embargo, se requiere de reforzar y promover esas nuevas competencias, dado que los dispositivos de lectura exigen otros comportamientos y actitudes para que esto se pueda llevar satisfactoriamente.

\section{DISCUSIÓN}

Analizar la lectura en los universitarios por medio de los conceptos de los habitus y las representaciones sociales implicó observar las acciones de los principales actores desde las prácticas cotidianas hasta la concreción de políticas educativas que realizamos quienes estamos involucrados con formación lectora en la universidad.

Se destaca que en los resultados existe una estrecha relación entre el capital cultural de los estudiantes y su capital económico, es decir que el estrato social al que pertenecen los estudiantes constituye parte importante para que ellos puedan no solo acceder sino también a utilizar de manera óptima el Internet, las bases de datos, bibliotecas digitales y otros acervos especializados acordes a las disciplinas en las que se están formando. Como se observó en la tabla 3 más del 40\% de los estudiantes en ambas unidades académicas no se consideran lectores, pese a que leen por horas tanto en formato impreso como en digital, además de ser usuarios frecuentes de internet. Los estudiantes leen más de lo que ellos mismos consideran. Esto como consecuencia de su percepción, ya que para ellos el hecho de no leer textos completos, información propiamente académica y/o en medios electrónicos ya suponen que eso no lo sea, porque no coincide con lo que se expresa en instituciones educativas y por los organismos encargados de medir los indicadores de la lectura. En este sentido los estudiantes reproducen una representación social hasta hace poco compartida por los académicos, que de manera velada o no, aún presentan resistencia a reconocer y valorar los nuevos soportes y sus formas de leer.

Aunado a eso, se observó que en las instituciones académicas se ha enseñado a leer, pero se ha puesto poco énfasis en la formación de lectores. Se observó también que quien no lee en medio impreso tampoco lo hace en medio digital, sin embargo que a la inversa si sucede. Como lo expresa Cordón $(2010,45)$ las nuevas prácticas de lectura “deben convivir” con esta auténtica 
revolución de los comportamientos culturales de las masas y apoyar a estas nuevas generaciones de lectores, cuyas habilidades tendrán que ser distintas, pues los textos digitales ya no son cerrados, ni lineales e intervienen otros factores como la hipertextualidad.

Cordón (2010) refiere datos de algunas investigaciones que se llevaron a cabo en España y en Francia entre 2009 y 2010. En estos países desarrollados no solo se da el acceso a la lectura digital, es un hecho tan cotidiano que ha propuesto nuevas formas de leer, de escribir y ha permeado la industria editorial; pues en dichos países ya se escribe literatura para leerse en dispositivos móviles con estructuras y herramientas para esos soportes. En los países desarrollados ya se ha superado la discusión que polariza lo impreso y lo digital.

En México y en Sonora cada vez se extiende más el acceso a leer en medios digitales, al menos en los grupos de jóvenes que han accedido a la universidad. Sin embargo, podemos decir que la reflexión no está en el mero acceso, sino en el uso que se les da a dichos recursos y su impacto el capital cultural de sus lectores, pues dicho capital no se potencia. En términos de Bourdieu (2009) podemos decir que aún no es un capital cultural incorporado porque su uso es incipiente, o instrumental. El 78.8\% de los estudiantes de esta investigación utilizan el internet para hacer sus tareas y solo entre el 10.5\% y el 12.2\% consultan libros (impresos o electrónicos) para ese fin, que suelen ser fuentes más especializadas. También se observa que, mientras el uso de las bases de datos aún es bajo, el 80.8\% de los estudiantes de la UABJ y el 56.3\% de la UAH utilizan las redes sociales de tres a cuatro horas al día.

A partir de este estudio se pone de manifiesto tres aspectos que consideramos relevantes:

1) Se reconoce la penetración de la lectura en formato digital entre los estudiantes universitarios y la sociedad en general y que confiere concepto de lectura un capital simbólico más ampliamente reconocido en el ámbito universitario.

2) La trascendencia del trabajo cotidiano de los docentes en las clases con los estudiantes, una labor, en apariencia imperceptible, pero que tiene un efecto importante en su formación lectora pues refuerza su gusto por leer, su capital cultural por medio del trabajo académico y de la inculcación ${ }^{7}$ y que contribuye a la trasmisión de habitus y representaciones sociales de la lectura.

\footnotetext{
${ }^{7}$ Se utiliza el término inculcación como el trabajo pedagógico infundir, transmitir mediante la socialización y la objetivación del discurso por medio del cual se confiere un a importancia o valor simbólico a prácticas y/o rituales. (Bourdieu, 2009)

Amaviz̨ca, Álvarezy Hernández doi: https://doi.org/10.36799/el.v2i1.51 Volumen 2, número 1, Año 2017, ISSN: $2448-5942$
} 
3) Se requiere aprovechar la tendencia hacia la lectura en soportes digitales para incorporarla como medios y estrategias en los diseños y rediseños curriculares de los programas educativos de las instituciones de educación superior y apoyar así la formación de lectores y los procesos de enseñanza- aprendizaje. También es importante que las instituciones fortalezcan su infraestructura en centros de cómputo, bibliotecas así como la conectividad a Internet, para que dichos servicios estén al alcance de todos los estudiantes sin importar su condición económica.

Esta investigación conduce a valorar la información cotidiana, de sentido común y a la lectura en medios digitales que se manifiesta en los habitus y las representaciones sociales. Dichas investigaciones y la incorporación de otras formas y dispositivos móviles para leer pueden contribuir para mejorar los sistemas educativos, desde sus proyectos estructurales hasta las estrategias utilizadas en las aulas. También conduce a valorar la importancia de la investigación social y educativa como actividad sustantiva del docente y fundamento para los diseños y rediseños curriculares.

Finalmente, este estudio nos llevó a reflexionar que entre la formalidad y la informalidad de leer en medio impreso o electrónico no debe haber un abismo infranqueable para que la lectura siga siendo una actividad cotidiana y amena, que aporte a la formación académica, profesional y humana de los estudiantes.

\section{BIBLIOGRAFÍA}

Bourdieu, Pierre. "Los tres estados del capital cultural". Sociológica, 2. 5. (1987), UAMAzcapotzalco.

Bourdieu, Pierre. Razones prácticas sobre la teoría de la acción. Barcelona: Anagrama, 1997.

Carlino, Paula. Escribir, leer y aprender en la universidad. Argentina: Fondo de Cultura Económica, 2005

Cassany, Daniel. Para ser letrados. España: Paidós, 2012.

Cassany, Daniel. Tras las líneas: sobre la lectura contemporánea. España: Anagrama, 2006.

Roger, Chartier. “¿Muerte o transfiguración del lector?” Revista de Occidente (2001): 72-86.

Amavižca, Alvarezy Hernández doi: https://doi.org/10.36799/el.v2i1.51 Volumen 2, número 1, Año 2017, ISSN: $2448-5942$ 
Cordón, José Antonio. De la lectura ensimismada a la lectura colaborativa: nuevas topologías de la lectura en el entorno digital. Salamanca, ES: Ediciones Universidad de Salamanca, 2010. ProQuest ebrary. Web. 15 Mar 2017.

Domingo, Juan. La letra muerta, tres diálogos virtuales sobre la realidad de leer. México: Océano, 2010.

Gutiérrez, Ariel. "E- Reading, la nueva revolución de la lectura: del texto impreso al cibertexto". Revista UNAM, 7. 5. (2006). Consultado el 27 de agosto de 2016.

http://www.revista.unam.mx/vol.7/num5/art42/may art42.pdf.

Hernández, Roberto., Fernández Carlos y Pilar Baptista. Metodología de la Investigación. México: McGraw Hill, 2006.

Ingarden, Roman., La comprensión de la obra de arte literaria. México: Universidad Iberoamericana, 2005.

Martínez, Piedad Cristina. "El método de estudio de caso: estrategia metodológica de la investigación científica". Pensamiento \& Gestión, 20. (2006): 165-193. Consultado el 10 de junio de 2016. http://www.redalyc.org/pdf/646/64602005.pdf

Moscovici, Serge. Prólogo. En W. H. Wagner. El discurso de lo cotidiano y el sentido común. México:Anthropos, 2011, pp 9-18.

Moscovici, Serge. Psicología social._Paidos, 1979.

OCDE. "Estudios económicos de la OCDE: México 2011". OECD Publishing (2011). Consultado el 10 de junio de2016. http://dx.doi.org/10.1787/9789264115934-es

Ruiz de Querol, Ricard y Jordi Buira, J. La sociedad de la información. Barcelona: Ebrary, 2012.

Universidad Estatal de Sonora, UES. Estadísticas anuales de biblioteca, 2016. 